\title{
Integrating LHCb workflows on HPC resources: status and strategies
}

\author{
Federico Stagni ${ }^{1, *}$, Andrea Valassi $^{2}$, and Vladimir Romanovskiy ${ }^{3}$ \\ ${ }^{1}$ CERN, EP Department, Geneva, Switzerland \\ ${ }^{2}$ CERN, IT Department, Geneva, Switzerland \\ ${ }^{3}$ NRC Kurchatov Institute", IHEP, Protvino, Russia
}

\begin{abstract}
High Performance Computing (HPC) supercomputers are expected to play an increasingly important role in HEP computing in the coming years. While HPC resources are not necessarily the optimal fit for HEP workflows, computing time at HPC centers on an opportunistic basis has already been available to the LHC experiments for some time, and it is also possible that part of the pledged computing resources will be offered as CPU time allocations at HPC centers in the future. The integration of the experiment workflows to make the most efficient use of HPC resources is therefore essential. This paper describes the work that has been necessary to integrate LHCb workflows at a specific HPC site, the Marconi-A2 system at CINECA in Italy, where LHCb benefited from a joint PRACE (Partnership for Advanced Computing in Europe) allocation with the other Large Hadron Collider (LHC) experiments. This has required addressing two types of challenges: on the software application workloads, for optimising their performance on a many-core hardware architecture that differs significantly from those traditionally used in WLCG (Worldwide LHC Computing Grid), by reducing memory footprint using a multi-process approach; and in the distributed computing area, for submitting these workloads using more than one logical processor per job, which had never been done yet in LHCb.
\end{abstract}

\section{Introduction}

LHCb is constantly looking for ways to opportunistically expand its distributed computing resources, beyond those pledged by the sites of the Worldwide LHC Computing Grid (WLCG). One way of doing so is by integrating High Performance Computing (HPC) supercomputers in the LHCb grid, managed via DIRAC [1] and its LHCb extension, LHCbDIRAC [2].

LHCb's interest in using HPC sites is mainly for running Monte Carlo (MC) simulation jobs. MC simulation jobs are, in fact, by far, the largest consumers of the LHCb share of WLCG compute resources (will be more than $90 \%$ in Run3). When it comes to distributed computing, the LHCb strategy is to use any new compute resources to run more MC simulation. The fraction of non-simulations jobs and CPU, in $\mathrm{LHCb}$, is small enough that we can rely, for them, on the currently existing pledged resources.

This paper describes the work that was performed in $\mathrm{LHCb}$ to be able to run MC simulation jobs on the Marconi-A2 HPC facility at CINECA in Bologna, Italy. An allocation

\footnotetext{
*e-mail: federico.stagni@cern.ch
} 
on this supercomputer became available to LHCb in mid-2019 within the context of a joint application of the Italian LHC community for a PRACE grant on this resource, as described more in detail in another presentation at this conference [3]. This work leveraged on the close collaboration between CINECA and CNAF, the Italian Tier- 1 site for WLCG, which is managed by the Istituto Nazionale di Fisica Nucleare (INFN) and is also located in Bologna.

Two different challenges had to be addressed to integrate the LHCb simulation workflow on the Marconi-A2 system, powered by many-core Intel Knights Landing (KNL) processors, with limited RAM memory per hardware thread: first, the LHCb MC software application, Gauss [4], had to be re-engineered to use multi-processing (MP) or multi-threading (MT) to have a lower memory footprint per thread; second, the LHCbDIRAC framework had to modified to be able to submit MP or MT jobs on batch queues, as this was the first time these types of jobs were used in LHCb distributed computing (even if this is expected to be the norm in the future, also for other types of workflows such as event reconstruction).

This paper is organized as follows. Section 2 describes the generic challenges for integrating LHCb computing workflows on HPC resources. Section 3 introduces the Marconi-A2 HPC at CINECA. Section 4 describes the work that was done to commission multi-processing applications in the Gauss simulation software. In section 5 the DIRAC project is briefly introduced, with a focus on the capabilities of the DIRAC Workload Management System. Section 6 gives more details about the distributed computing challenges and solutions for LHCbDIRAC on Marconi-A2. Finally, conclusions are given in Section 7.

\section{Challenges of High Performance Computer systems}

Using HPC facilities, in LHCb but more generally for any HEP experiment, poses two rather distinct types of challenges:

- Software architecture challenges: the compute power of HPC supercomputers may come from a range of different processor architectures, including multi-core and many-core x 86 CPUs, non-x86 CPUs (ARM, Power9) and accelerators (GPUs, FPGAs). For an LHC experiment, being able to efficiently exploit these resources may require significant changes to its software applications, which are generally designed for the setup of a traditional WLCG worker node, based on an x86 CPU with at least 2 GB RAM available per hardware thread. In addition, HPCs provide extremely fast inter-node connectivity, often used for parallel processing using MPI, while most HEP software applications generally use the individual nodes independently of one another, as if HPCs were just very large clusters.

- Distributed computing challenges: HPC sites usually have strict site policies for security reasons and may therefore be characterized by limited or absent external connectivity, adhoc operating systems, limited storage on local disks, restrictive policies for user authentication/authorization and for batch queues. This differs from the configuration of traditional sites in WLCG, which provide full access to remote services like the CernVM File System (CernVM-FS, often abbreviated CVMFS[5]) for software installation, uniform operating systems and the capability to use user-level virtualization.

More generally, unlike WLCG sites which provide a relatively uniform computing environment, HPC centers may differ significantly from one another. Some HPCs are easier to exploit than others, e.g. LHCb already uses Piz Daint at CSCS [6], which looks like a traditional Grid site providing a cluster of nodes powered by x 86 CPUs. The collaboration of the experiments with the local system administrators and performance experts is in any case essential to address the specific issues of each HPC center, and has proved to be mutually beneficial.

All in all, HPCs are not the most natural fit for HEP computing today. Because of the large amounts of resources dedicated to scientific computing that are currently deployed at HPC 
centers now, and of their predicted further increase in the future, it is however essential that LHC experiments continue to work on adapting their software and computing infrastructures to be able to efficiently exploit these resources in the near future.

\section{Marconi-A2: a KNL based partition of the CINECA supercomputer}

Marconi [7] is a supercomputer at CINECA, available for the Italian and European research community. Currently ranked number 19 in the top500.org list [8], Marconi provides its compute capacity through several independent partitions. The Marconi-A2 partition, which has been used for the work described in this paper, consists of nodes equipped with one Xeon Phi 7250 (KNL) at $1.4 \mathrm{GHz}$, with $96 \mathrm{~GB}$ of on board RAM. This is an x86 many-core CPU with 68 physical cores, supporting 4-way hyperthreading. Keeping into account that approximately $10 \mathrm{~GB}$ of memory are reserved for the $\mathrm{O} / \mathrm{S}$, this means that just over $300 \mathrm{MB}$ of RAM are available per hardware thread if all 272 threads are used. This is much lower than the 2 GB (or more) per thread available at WLCG sites, motivating the effort to implement MP and MT approaches in the Gauss software, as described in the next Section 4.

In January 2020, most of the A2 partition (which included 3600 nodes in 2019) was switched off, to upgrade Marconi's compute capacity by replacing the KNLs in Marconi-A2 by the GPUs in a new Marconi100 partition. Some of the KNLs in A2 are however still available at the time of writing in March 2020, and will be used for our LHCb work until the end of the granted allocation. While the efficient exploitation of KNLs already required some software development effort, as described in the next section, it should be noted that the work we describe could not have been performed on the GPU-based partition, as neither event generation nor detector simulation are yet possible in the LHCb software on GPUs.

The default computing environment on Marconi is also quite different from that normally found at WLCG sites. Thanks to the excellent collaboration between the Italian experiment contacts and the site managers and sysadmins at CINECA and CNAF, many essential ad-hoc changes were deployed for the LHC experiments [3]. In particular: CVMFS mounts and Squids were provided; external outgoing networking was partially opened at CINECA, with routing active to the IP ranges of CERN, FermiLab and CNAF; the Singularity [9] container management tool was deployed; a HTCondor-CE (the HTCondor Computing Element) was allowed on a CINECA edge node, for submitting jobs to the internal SLURM batch system connected to Marconi-A2. The only change that was needed to allow MP/MT LHCb MC simulation workflows on Marconi-A2 was therefore the implementation of MP/MT job submission in LHCbDIRAC, as described in Section 6.

\section{Multi-process LHCb MC simulation on Marconi-A2: GaussMP}

The LHCb software for running Monte Carlo simulations, Gauss [4], is used for both event generation [10] and detector simulation, the latter using internally the Geant4 [11] simulation toolkit. Currently, MC simulation jobs in LHCb execute both steps sequentially on the same worker node, therefore they need basically no input data (only a configuration file). This implies that, unlike workflows such as event reconstruction or stripping, the management of input data files is not an issue for MC simulation. In addition, both event generation and detector simulation software applications are compute-intensive, rather than I/O-intensive. Optimising their performance is mainly a problem of an efficient use of CPU and memory.

Until recently, LHCb has only used a single-process (SP), single-threaded (ST), version of the Gauss software for all of its MC simulation productions. Only x86 architectures are currently supported, although ports to ARM have been worked on. The typical memory 
footprint of these applications, around 1.4 GB [12], has so far not been an issue on traditional WLCG nodes, where 2 GB per thread are available. On many-core CPUs like the KNL used in Marconi-A2, however, these workflows are very inefficient, because the limited memory available per thread (300 MB if 4 threads are used on each of the 68 physical cores of a KNL, or $600 \mathrm{MB}$ if only 2 threads are used) effectively limits the maximum number of SP/ST Gauss instances that can be executed simultaneously, i.e. the number of KNL threads that can be filled. To reduce the memory footprint per thread and be able to fill a larger number of KNL threads, multi-processing (MP) or multi-threading (MT) approaches are needed.

In the long term, the LHCb solution will be to base its MC simulations on Gaussino [13], a MT implementation of Gauss. In spite of its very fast recent progress, however, Gaussino was still not ready for meaningful productions or tests when the Marconi-A2 allocation started. As a temporary solution, the software work targeting the Marconi A2 timescales focused on the test and commissioning of GaussMP, a MP-based version of Gauss. This leveraged on GaudiMP [14, 15], a MP version of the LHCb event processing framework Gaudi, which already existed but had never been used in production.

The software work on GaussMP had two main aspects: extensive functional testing and bug-fixing, and performance testing and optimization. Functional testing and bug-fixing essentially targeted, and achieved, a validation of results requiring identical results in the SP and MP versions of Gauss, when simulating the same set of events, starting from the same random number seeds. In other words, rather than requiring a physics validation of results by comparing physics distributions within MC statistical errors, the MP software was validated by requiring that some event-by-event properties (numbers of particles and vertices etc.) should be the same in MP and SP applications. This implied careful checks in both the event generation and detector simulation steps of the application.

Performance testing and optimization essentially consisted in running several identical copies of an application on a given worker node, in SP mode or using different MP configurations, to understand which configuration maximises the total throughput of the entire node, i.e. the number of events processed per unit wall-clock time. Memory usage was also monitored to provide an interpretation of throughput results. The tests were performed both on a reference node at CERN, using a traditional hardware setup based on two multi-core Haswell CPUs with 2 GB per hardware thread (2x8 physical cores with 2-way hyperthreading and 64 GB RAM), and on a KNL node from Marconi-A2 (68 physical cores with 4-way hyper-threading and 96 GB RAM).

The results of these tests are shown in Fig. 1 for the reference node at CERN and in Fig. 2 for the KNL on Marconi-A2. The memory footprint of the physics process used for this specific test (event generation and detector simulation of $B^{+} \rightarrow J / \psi K^{+}$production, including spillover from minimum bias production in adjacent collisions) is around $900 \mathrm{MB}$ per process/thread in SP mode. On the reference node, with 64 GB RAM, 32 instances of an SP application can be used to fill all 32 threads, and this is the configuration providing maximum throughput (6.0 events per minute): several MP configurations provide similar, but slightly lower, integrated throughput (for instance, 5.8 events per minute for 8 instances of GaussMP with 4 processes each), because of the overhead involved in the extra processes used by GaussMP. On the KNL node, however, at most 85 SP instances can be launched, because some processes are killed by the out-of-memory monitor if more instances are launched. Using GaussMP results in a lower memory footprint per hardware thread, allowing a larger number of KNL threads to be filled: in particular, the maximum throughput on the KNL is achieved when 8 GaussMP application instances are executed in parallel, each using 17 processes. This corresponds to using 2 hardware thread per KNL core (i.e. 136 in total), not 4: many failures are observed when trying to use 4 threads per KNL core (i.e. 272 in total). 

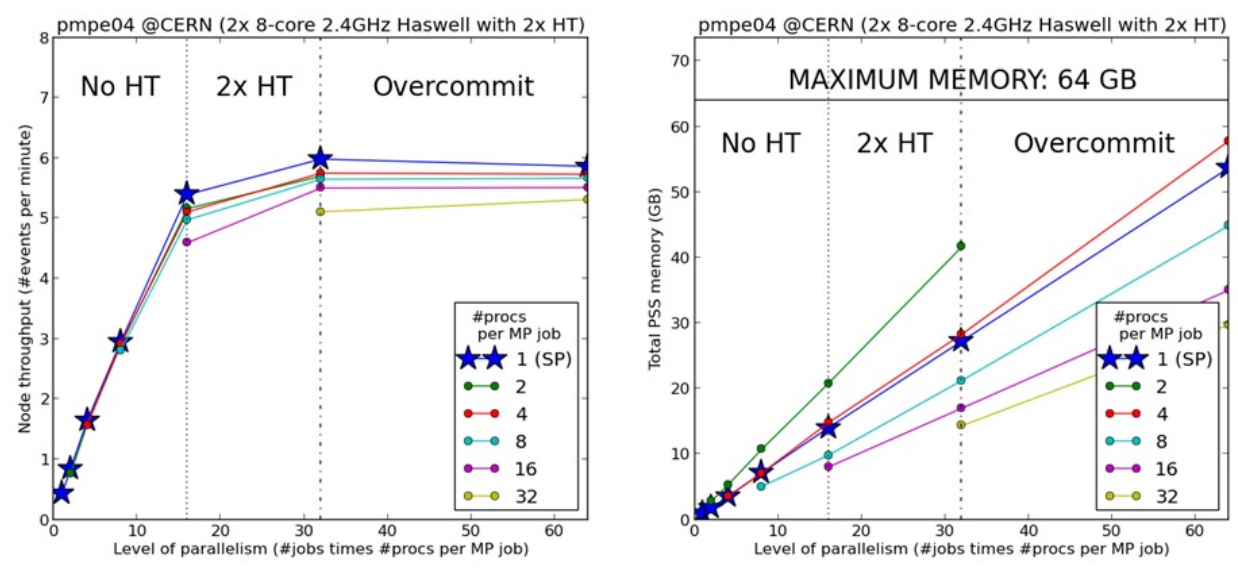

Figure 1. Gauss and GaussMP: throughput and memory on the reference Haswell node at CERN (2x8 physical cores with 2-way hyperthreading and 64 GB RAM).
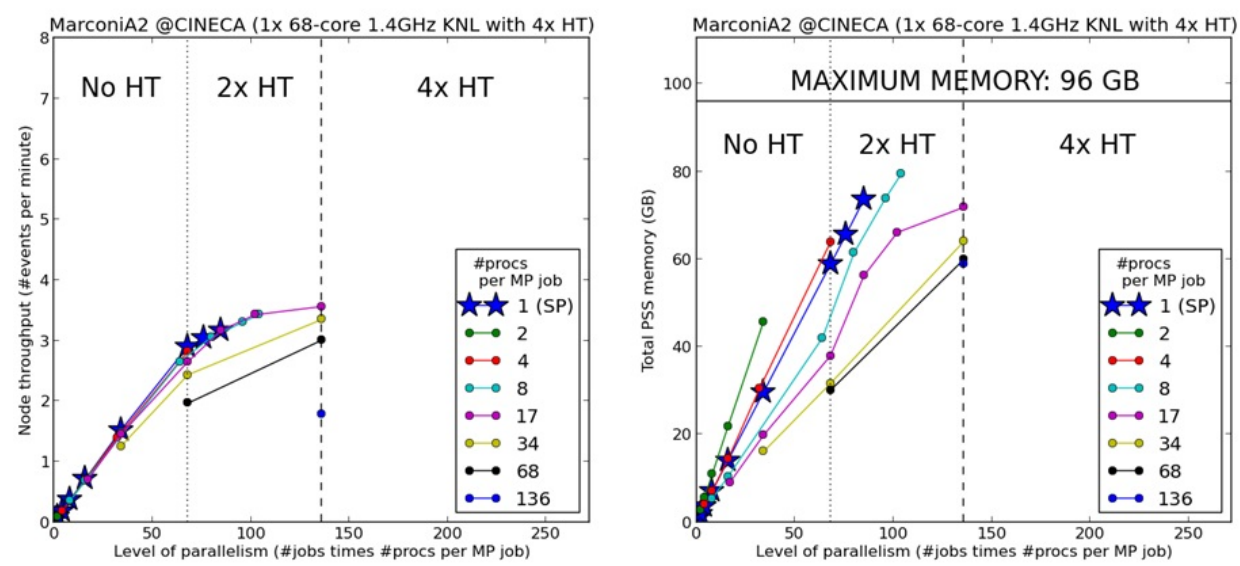

Figure 2. Gauss and GaussMP: throughput and memory on a Marconi-A2 KNL node at CINECA (68 physical cores with 4-way hyper-threading and 96 GB RAM).

The highest GaussMP throughput achieved on the KNL (3.6 events per minute) is only moderately higher $(\sim 15 \%)$ than that achieved using SP Gauss (3.2 events per minute), because the forking strategy used does not optimize the use of copy-on-write to minimize the memory footprint. Currently, new worker processes are forked after job initialization but before the first event $[14,15]$, where the magnetic field map is read from disk [16]; forking workers after processing the first event would make it possible to share a larger amount of memory across workers and reduce the overall memory footprint, as recently demonstrated by ATLAS in their new AthenaMP forking strategy [17]. Looking forward, however, LHCb software efforts in the simulation area will focus on Gaussino, the long-term MT solution, rather than on GaussMP, the temporary MP solution.

It is also interesting to note that, in absolute terms, the throughput per thread achieved in the configuration maximising the total node throughput is a factor 7 lower on the KNL ( 0.026 events per minute per core, for 3.6 events per minute on 136 threads) than on the reference Haswell node ( 0.188 events per minute per thread, for 6.0 events per minute on 32 threads). This can only be partly explained in terms of the lower clock speed of the KNL cores, and is probably also due to the memory access patterns of the Gauss application on the two architectures, but no specific studies have been performed to understand this better. 


\section{The DIRAC project}

DIRAC [1] is a software framework that enables communities to interact with distributed computing resources. It builds a layer between users and resources, hiding diversities across computing, storage, catalog, and queuing resources. DIRAC has been adopted by several HEP and non-HEP experiment communities [18], with different goals, intents, resources and workflows: it is experiment agnostic, extensible, and flexible [19]. LHCb uses DIRAC for managing all its distributed computing activities. DIRAC is an open source project, which was started around 2002 as an LHCb project. Following interest of adoption from other communities its code was made available under open licence in 2009. Now, it is hosted on GitHub [20] and is released under the GPLv3 license.

The DIRAC Workload Management System (WMS) is in charge of exploiting distributed computing resources. In other words, it manages jobs, and pilot jobs [21] (from here on simply called "pilots"). The emergence of new distributed computing resources (private and commercial clouds, High Performance Computing clusters, volunteer computing, etc) changed the traditional landscape of computing for offline processing. It is therefore crucial to provide a very versatile and flexible system for handling distributed computing (production and user data analysis). If we restrict for a moment our vision to LHC experiments, and we analyze the amount of CPU cycles they used in the last year, we can notice that all of them have consumed more CPU-hours than those official reserved (pledged) to them by WLCG. Each community found ways to exploit "opportunistic", i.e. non-pledged, compute resources (CPUs, or even GPUs). Such resources may be private to the experiment (e.g. the "online" computing farm - often simply called "High Level Trigger" farm) or public; resources may sometimes be donated free of charge, like in the case of volunteer computing, or not, like public commercial cloud providers. Integrating non-grid resources is common to all communities that have been using WLCG in the past, and still do. Communities that use DIRAC want to exploit all possible CPU or GPU cycles. Software products like DIRAC aim to make this easy, and the DIRAC pilot is the federator of each and every computing resource. With DIRAC, transparent access to the computing resources is realized by implementing the pilot model.

\section{Distributed computing challenges on Marconi-A2: fat nodes}

The integration of new compute resources into the LHCb distributed computing framework, based on DIRAC, is generally an easy task when: first, worker nodes (WNs) have outbound network connectivity; second, the LHCb CVMFS endpoints are mounted on the WNs; and, third, the WN O/S is an SLC6 (Scientific Linux CERN 6) or CC7 (CERN CentOS 7) compatible flavor, or Singularity containers are available. As discussed in Sec. 2, none of these conditions would normally be satisfied on Marconi, but all three were eventually met on Marconi-A2 specifically for the LHC experiments, thanks to the good collaboration between the experiment contacts and the site managers.

As a consequence, on the distributed computing side, the main challenge $\mathrm{LHCb}$ had to address was that each job slot provided by the HTCondorCE represents a whole KNL node, with 68 physical cores and up to 4 hardware threads per core, i.e. a total of nproc $=272$ logical processors (assuming that 4-way hyper-threading is enabled). Rather than implementing a quick ad-hoc solution for Marconi-A2, this was addressed in DIRAC by developing a generic mechanism for managing "fat nodes", as shown schematically in Figs. 3 and 4. In DIRAC terminology, this problem, of how to subdivide a fat node and allocate its resources to several jobs, is simply called "matching". It is worth noting that this was never done in LHCb before, as only jobs running SP/ST software workloads and using a single logical processor were used. 


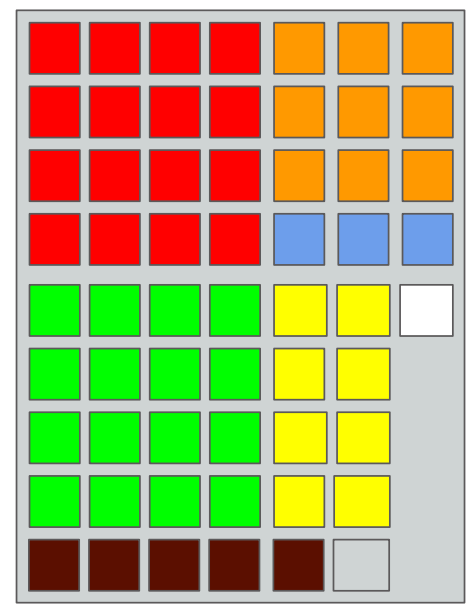

Figure 3. Allocating jobs within a fat node: the DIRAC pilot, when using the Pool inner Computing Element, realizes a de-facto partitioning of the node, and parallel jobs matching. The figure above shows a theoretical allocation of jobs to logical processors, with each box representing a logical processor. Each color represents a different application.

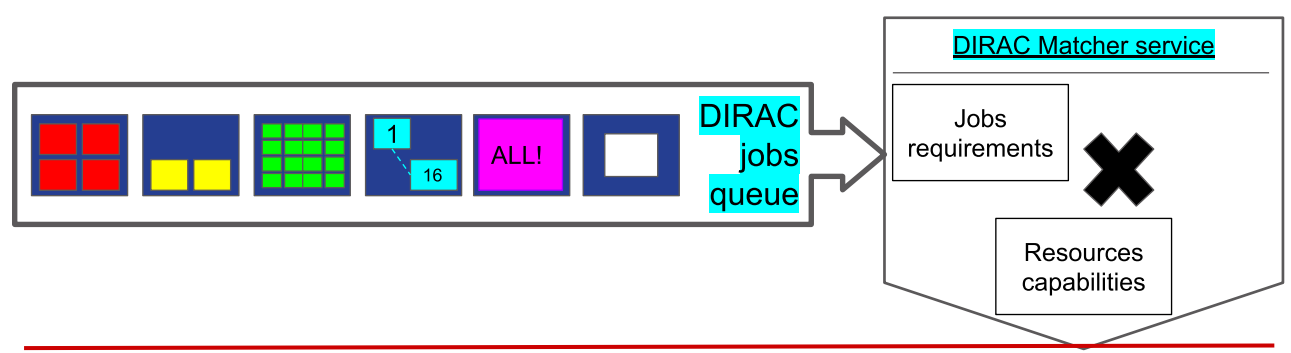

Resources (1 pilot per box)
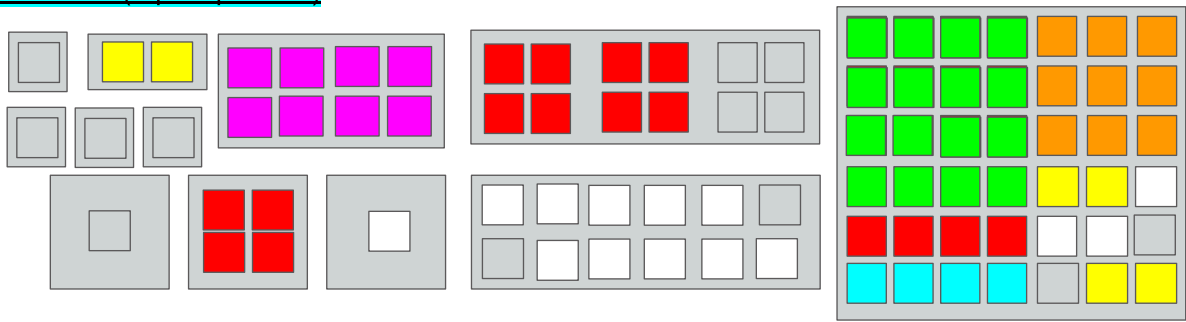

Figure 4. Job matching: matching job requirements to computing resources.

For a proper job allocation, DIRAC needs to "partition" the node for optimal memory and throughput (and maybe only use a subset of the logical processors). For this to happen, we have developed the Pool "inner" Computing Element, with which it is possible to execute parallel jobs matching. In the following, by "processor" we mean a "logical processor" (whose number is nproc in total); by "single-processor" jobs we mean single-threaded, single-process software application workloads, requiring a single logical processor, while by "multi-processor" job we mean a software application workload that uses more than one logical processor, whether the application is implemented using a multi-process approach (like GaussMP) or a multi-threaded approach (like Gaussino), or a combination of both. 
From a user's perspective, it is possible to describe the jobs precisely enough to satisfy all use cases below:

- certain jobs may be able to run only in single-processor mode

- certain jobs may be able to run only in multi-processor mode (i.e., they need at least 2 logical processors)

- certain multi-processor jobs may need a fixed amount of logical processors (in our specific case on Marconi-A2, we chose to submit only GaussMP jobs, at most 8 simultaneously, using 17 logical processors per job, to maximize the whole node throughput)

- certain jobs may be able to run both in single-processor or multi-processor mode, depending on what is possible on the WN/Queue/CE

- for certain jobs we may want to specify a maximum number of processors to use

At the same time, from a resource provider's perspective, it is possible to describe CEs and Queues precisely enough to satisfy all use cases below:

- may give their users the possibility to run on their resources:

- only single processor jobs

- both single and multi processor jobs

- may ask their users to distinguish clearly between single and multi processor jobs

- may need to know the exact number of processors a job is requesting

- may ask for only "wholeNode" jobs

\section{Summary and outlook}

In summary, both of the challenges involved in the integration of LHCb MC simulation workflows on the Marconi-A2 HPC have been addressed: a multi-process version of the Gauss software framework with reduced memory footprint per thread has been commissioned, and the functionality of managing fat nodes has been added to the LHCdDIRAC distributed computing framework and has been successfully tested in a dedicated certification environment. At the time of writing in March 2020, however, the new LHCbDIRAC functionality has not yet been deployed in production, because of the timescales involved in the LHCb software release process. This is the reason why no results of production use of Marconi A2 by LHCb for MC simulation using GaussMP are shown in this paper. As soon as the new LHCbDIRAC is released within the next few weeks, however, the remaining LHCb allocation on the MarconiA2 KNLs will be used to launch the first production jobs of MC simulation, using software workflows based on GaussMP, as well as using Gaussino if available on time.

More generally, this effort at integrating a new HPC resource into the LHCb software and computing was extremely valuable. On the software application side, it was useful to highlight some of the challenges ahead in the use of non-traditional compute architectures (which may well be GPUs in the not-so distant future). On the distributed computing side, it was useful to pave the way to the more routine use of multi-threaded software applications on the grid, which will soon become the norm. Last but not least, the collaboration with the other LHC experiments and with the local site managers and sysadmins at CINECA and CNAF was an essential ingredient of this effort, and a pleasant and fruitful experience for which we thank them, and that we look forward to repeating in the future. 


\section{References}

[1] F. Stagni et al., DIRACGrid/DIRAC (2018). https://doi.org/10.5281/zenodo.1451647

[2] LHCb Coll., LHCbDIRAC (2018). https://doi.org/10.5281/zenodo.1451768

[3] T. Boccali et al., Extension of the INFN Tier-1 on a HPC system, to appear in Proc. CHEP2019, Adelaide (2019). https://indico.cern.ch/event/773049/contributions/3474805

[4] M. Clemencic et al., The LHCb Simulation Application, Gauss: Design, Evolution and Experience, Proc. CHEP2010, Taipei, J. Phys. Conf. Ser. 331, 032023 (2011). https://doi.org/10.1088/1742-6596/331/3/032023

[5] J. Blomer et al., Distributing LHC application software and conditions databases using the CernVM file system, Proc. CHEP2010, Taipei, J. Phys. Conf. Ser. 331, 042003 (2011). https://doi.org/10.1088/1742-6596/331/4/042003

[6] F. G. Sciacca, S. Haug et al., ATLAS and LHC computing on CRAY, Proc. CHEP2016, San Francisco, J. Phys. Conf. Ser. 898, 082004 (2017). https://doi.org/10.1088/17426596/898/8/082004

[7] Marconi at CINECA, http://www.hpc.cineca.it/hardware/marconi

[8] top500 rankings as of November 2019, https://www.top500.org/lists/2019/11

[9] G. M. Kurtzer, V. Sochat, M. W. Bauer, Singularity: Scientific containers for mobility of compute, PLoS ONE 12, e0177459 (2017). https://doi.org/10.1371/journal.pone.0177459

[10] I. Belyaev et al., Handling of the generation of primary events in Gauss, the LHCb simulation framework, Proc. CHEP2010, Taipei, J. Phys. Conf. Ser. 331, 032047 (2011). https://doi.org/10.1088/1742-6596/331/3/032047

[11] S. Agostinelli et al., Geant4 - a simulation toolkit, NIM A 506, 250 (2003). https://doi.org/10.1016/S0168-9002(03)01368-8

[12] G. Corti et al., Computing performance of the LHCb simulation, 24th Geant4 Collaboration Meeting, JLAB (2019). https://indico.cern.ch/event/825306/contributions/3565311

[13] D. Muller, Gaussino - a Gaudi-based core simulation framework, to appear in Proc. CHEP2019, Adelaide (2019). https://indico.cern.ch/event/773049/contributions/3474740

[14] N. Rauschmayr, A. Streit, Preparing the Gaudi framework and the DIRAC WMS for multicore job submission, Proc. CHEP2013, Amsterdam, J. Phys. Conf. Ser. 513, 052029 (2014). https://doi.org/10.1088/1742-6596/513/5/052029

[15] N. Rauschmayr, Optimisation of LHCb applications for multi- and manycore job submission, CERN-THESIS-2014-242 (2014). https://cds.cern.ch/record/1985236

[16] A. Valassi, S. Muralidharan, Trident analysis of the LHCb GEN/SIM workload from the benchmarking suite, System performance modelling WG meeting, CERN (2019). https://indico.cern.ch/event/772026

[17] J. Elmsheuser et al., ATLAS Grid Workflow Performance Optimization, Proc. CHEP2018, Sofia, EPJ Web of Conf. 214, 03021 (2019). https://doi.org/10.1051/epjconf/201921403021

[18] F. Stagni et al., DIRAC in Large Particle Physics Experiments, Proc. CHEP2016, San Francisco, J. Phys. Conf. Ser. 898, 092020 (2017). https://doi.org/10.1088/17426596/898/9/092020

[19] S. Camarasu-Pop et al., Exploiting GPUs on distributed infrastructures for medical imaging applications with VIP and DIRAC, Proc. MIPRO2019, Opatija (2019). https://doi.org/10.23919/mipro.2019.8757075

[20] DIRAC project GitHub repository, https://github.com/DIRACGrid

[21] F. Stagni et al., DIRAC universal pilots, Proc. CHEP2016, San Francisco, J. Phys. Conf. Ser. 898, 092024 (2017). https://doi.org/10.1088/1742-6596/898/9/092024 